\title{
Noether theory for Birkhoffian systems with nabla derivatives
}

\author{
Chuanjing Songa ${ }^{\mathrm{a}}$, Yi Zhang ${ }^{\mathrm{b}, *}$ \\ ${ }^{a}$ College of Science, Nanjing University of Science and Technology, Nanjing 210094, P. R. China. \\ ${ }^{b}$ College of Civil Engineering, Suzhou University of Science and Technology, Suzhou 215009, P. R. China.
}

Communicated by D. Baleanu

\begin{abstract}
There are discrete phenomena which happen only on discrete time or hold discrete space structures such as economy series, population dynamics et al.. Then there is a tool needed for these discrete issues or applications. Time scale is one of the useful tools to solve some discrete problems. In this paper, time scale is used to establish discrete Pfaff-Birkhoff principle and achieve discrete Birkhoff equations, discrete Noether identity and discrete conserved quantity for the discrete Birkhoffian system. Firstly, Birkhoff equations, Noether identity and Noether theorem with nabla derivatives on time scales are investigated by using the isochronous variational principle. Secondly, some special cases, especially the discrete Birkhoffian system are discussed. Thirdly, another method, i.e., the duality principle is introduced for the Birkhoffian system on time scales. And finally, an example is given to illustrate the results and methods. (C) 2017 All rights reserved.
\end{abstract}

Keywords: Noether theorem, Birkhoffian system, time scale, duality principle, isochronous variational principle. 2010 MSC: 34N05, 39A12, 49K05.

\section{Introduction}

In 1973, Turrittin in [68], pointed out: "On becoming familiar with difference equations, I was in hopes that the theory of difference equations could be brought completely abreast with that for ordinary differential equations." Much earlier than Turrittin, Bell in 1937 in [20], once mentioned: "A major task of mathematics today is to harmonize the continuous and the discrete, to include them in one comprehensive mathematics, and to eliminate obscurity from both." Under this kind of background, Hilger introduced the definition of time scale in his $\mathrm{PhD}$ thesis in 1988, see [41]. A time scale is an arbitrary nonempty closed subset of real numbers. From the definition, the continuous analysis can be gotten when the time scale is the set of real numbers, and the discrete analysis can be gotten when the time scale is the set of integers. However, since there are many other time scales than just the sets of real numbers and integers, one can get much more results. Therefore, unification and extension are two main features of time scales. Time scale calculus mainly refers to two kinds of calculus: time scale delta calculus and time scale nabla calculus, both of them have received a lot of attention and some results on time scales have been obtained, we refer the readers, for instance, to [5, 6, 8, 10, 15-17, 25, 26, 30, 34, 42, 52-54]. In addition, the theory of time scales has also been applied to practical problems such as the optimal control problems, see $[17,43,78]$, and some problems on physics and economics, see $[4,8,27]$.

\footnotetext{
*Corresponding author

Email addresses: songchuanjingsun@126.com (Chuanjing Song), zhy@mail.usts .edu . cn (Yi Zhang)
} 
It is worth mentioning that some basic properties such as Laplace transform and existence of solutions of difference equations on time scales have been systemically discussed [3,28]. In order to consider the memory effects, References $[1,9,11]$ studied the fractional difference on time scales, which gives a useful tool for discrete fractional modelling. Some properties of fractional difference have been studied $[1,2,9,31,39,40,44]$, such as Laplace transform [44], existence and stability [31], initial value problems [9], boundary value problems [39, 40] and so on. Recently, fractional differences which become more and more popular have been used to study the diffusion over random media of discrete structures [73], discrete fractional chaos $[45,69-71,74,75]$, discrete fractional control $[59,60]$ and so forth.

Noether theory was introduced by Noether in 1918 during the process of getting the solutions of differential equations in [61]. Noether symmetry means the invariance of Hamilton action under the infinitesimal transformations of the time and the coordinates. Actually, it is hard to obtain analytical solutions for dynamic equations of motion for constrained mechanical systems. However, we can get conserved quantities, which can help reduce the degrees of freedom of the equations of motion, through the Noether symmetry. Conserved quantities for constrained mechanical systems can not only simplify the dynamical problems, but also reveal the internal physical regularity to some extent. Hence, it is of great significance to study. Up to now many results about Noether theory have been obtained, such as Noether theory based on fractional models, see [7, 35, 80, 81]; Noether theory with time delay, see [46, 77]; Noether theory for nonlinear dynamical systems, see [36, 82]; as well as Noether theory for fractional systems of variable order, see $[62,76]$. Recently, Noether theory was extended to time scales, see for instance, $[18,29,50,51,55,63,79,83]$.

However, most of the results about Noether theory on time scales just referred to Lagrangian system and Hamiltonian system. There is another more general mechanical system, i.e., Birkhoffian system.

Birkhoffian mechanics, whose quintessence is the Birkhoff equations and Pfaff-Birkhoff principle is a generalization of Hamiltonian mechanics, we refer to [24, 64]. Hamilton canonical equations keep the same forms under canonical transformations, and become the Birkhoff equations under non-canonical transformations. Therefore, Hamilton canonical equations are special cases of Birkhoff equations. Hamilton principle is also a special case of the Pfaff-Birkhoff principle. Hence, the theory of Birkhoffian mechanics can be applied to Hamiltonian mechanics, Lagrangian mechanics and Newtonian mechanics, as well as general holonomic and nonholonomic mechanics, see [57]. Besides, Birkhoffian mechanics is also applicable to statistical mechanics, quantum mechanics, biological physics, hadron physics, atomic and molecular physics and engineering, and other fields, see [64]. Galiullan in 1989 said that Birkhoffian mechanics is an important developmental direction of modern analytical mechanics in [37]. And in 1996, the theoretical framework of Birkhoffian mechanics was established by Mei et al. in [57]. Since then, scholars continue to study Birkhoffian mechanics deeply, we refer the readers for instance to [32, 33, 47$49,56,58,65-67,76,77,80,81]$.

Hence, in this paper, we intend to study Noether theory for Birkhoffian systems on time scales with nabla derivatives. Two methods are used: the isochronous variational principle and the duality principle. In Section 2, some necessary definitions and properties about time scale calculus and the duality approach are reviewed. In Section 3, the isochronous variational principle is used to study Noether theory for Birkhoffian systems on time scales with nabla derivatives, where Pfaffian variational problem on time scales with nabla derivatives is presented for the first time, the Birkhoff equations and the Noether theorem on time scales with nabla derivatives are derived, and some special cases, especially the discrete Birkhoffian system, of the main results are also discussed. The duality principle is used in Section 4 , in which Noether equations, Noether identity and conserved quantity for Birkhoffian systems on time scales with nabla derivatives are achieved again. Finally, an example is given to illustrate the results in Section 5 .

\section{Preliminaries}

We assume that the reader is familiar with the time scale calculus in [33]. Here we mainly review some necessary results about duality from [30]. 
If $\mathbb{T}$ is an arbitrary time scale, we denote $\mathbb{T}^{*}=\{s \in \mathbb{R} \mid-s \in \mathbb{T}\}$ as the dual time scale of $\mathbb{T}$, and the corresponding dual objects of $\mathbb{T}^{*}$ are $\hat{\sigma}, \hat{\rho}, \hat{\mu}$ and $\hat{v}$.

Definition 2.1. Given a function $f: \mathbb{T} \rightarrow \mathbb{R}$ defined on a time scale $\mathbb{T}$, we define the dual function $f^{*}: \mathbb{T}^{*} \rightarrow \mathbb{R}$ by $f^{*}(s)=f(-s)$ for all $s \in \mathbb{T}^{*}$.

Proposition 2.2. Let $f: \mathbb{T} \rightarrow \mathbb{R}, a, b \in \mathbb{T}, a<b$, then

(1) $\left(\mathbb{T}^{\mathrm{K}}\right)^{*}=\left(\mathbb{T}^{*}\right)_{\mathrm{K}}$ and $\left(\mathbb{T}_{\mathrm{K}}\right)^{*}=\left(\mathbb{T}^{*}\right)^{\mathrm{K}}$;

(2) $([\mathrm{a}, \mathrm{b}])^{*}=[-\mathrm{b},-\mathrm{a}]$ and $\left([\mathrm{a}, \mathrm{b}]^{\mathrm{K}}\right)^{*}=[-\mathrm{b},-\mathrm{a}]_{\mathrm{K}} \subseteq \mathbb{T}^{*}$;

(3) $\hat{\sigma}(s)=-\rho(-s)=-\rho^{*}(s)$ and $\hat{\rho}(s)=-\sigma(-s)=-\sigma^{*}(s)$ for all $s \in \mathbb{T}^{*}$;

(4) $\hat{\mu}(\mathrm{s})=\nu^{*}(\mathrm{~s})$ and $\hat{v}(\mathrm{~s})=\mu^{*}(\mathrm{~s})$ for all $\mathrm{s} \in \mathbb{T}^{*}$;

(5) $\mathrm{f} \in \mathrm{C}_{\mathrm{rd}}$ (resp.f $\left.\in \mathrm{C}_{\mathrm{ld}}\right) \Leftrightarrow \mathrm{f}^{*} \in \mathrm{C}_{\mathrm{ld}}\left(\right.$ resp. $\mathrm{f}^{*} \in \mathrm{C}_{\mathrm{rd}}$ ), $\mathrm{f} \in \mathrm{C}_{\mathrm{rd}}^{1}$ (resp.f $\left.\in \mathrm{C}_{\mathrm{ld}}^{1}\right) \Leftrightarrow \mathrm{f}^{*} \in \mathrm{C}_{\mathrm{ld}}^{1}$ (resp. $\mathrm{f}^{*} \in \mathrm{C}_{\mathrm{rd}}^{1}$ );

(6) if $\mathrm{f}$ is delta (resp. nabla) differentiable at $\mathrm{t}_{0} \in \mathbb{T}^{\mathrm{K}}$ (resp. $\mathrm{t}_{0} \in \mathbb{T}_{\mathrm{K}}$ ), then $\mathrm{f}^{*}$ is nabla (resp. delta) differentiable at $-\mathrm{t}_{0} \in\left(\mathbb{T}^{*}\right)_{\mathrm{K}}\left(\right.$ resp. $\left.-\mathrm{t}_{0} \in\left(\mathbb{T}^{*}\right)^{\mathrm{k}}\right)$, and

$$
\begin{gathered}
\mathrm{f}^{\triangle}\left(\mathrm{t}_{0}\right)=-\left(\mathrm{f}^{*}\right)^{\hat{\nabla}}\left(-\mathrm{t}_{0}\right) \quad\left(\text { resp. } \mathrm{f}^{\nabla}\left(\mathrm{t}_{0}\right)=-\left(\mathrm{f}^{*}\right)^{\hat{\Delta}}\left(-\mathrm{t}_{0}\right)\right), \\
\mathrm{f}^{\triangle}\left(\mathrm{t}_{0}\right)=-\left(\left(\mathrm{f}^{*}\right)^{\hat{\nabla}}\right)^{*}\left(\mathrm{t}_{0}\right) \quad\left(\text { resp. } \mathrm{f}^{\nabla}\left(\mathrm{t}_{0}\right)=-\left(\left(\mathrm{f}^{*}\right)^{\hat{\Delta}}\right)^{*}\left(\mathrm{t}_{0}\right)\right), \\
\left(\mathrm{f}^{\triangle}\right)^{*}\left(-\mathrm{t}_{0}\right)=-\left(\mathrm{f}^{*}\right)^{\hat{\nabla}}\left(-\mathrm{t}_{0}\right) \quad\left(\text { resp. }\left(\mathrm{f}^{\nabla}\right)^{*}\left(-\mathrm{t}_{0}\right)=-\left(\mathrm{f}^{*}\right)^{\hat{\Delta}}\left(-\mathrm{t}_{0}\right)\right) ;
\end{gathered}
$$

(7) $\int_{a}^{b} f(t) \triangle t=\int_{-b}^{-a} f^{*}(s) \hat{\nabla} s, \int_{a}^{b} f(t) \nabla t=\int_{-b}^{-a} f^{*}(s) \hat{\Delta} s$.

Lemma 2.3 ([54]). Let $\mathrm{g} \in \mathrm{C}_{\mathrm{ld}}, \mathrm{g}:[\mathrm{a}, \mathrm{b}] \rightarrow \mathbb{R}$, if

$$
\int_{a}^{b} g(t) \eta^{\nabla}(t) \nabla t=0
$$

holds for all $\eta \in C_{\text {ld }}^{1}$ with $\eta(a)=\eta(b)=0$, then

$$
g(t)=c, \quad t \in[a, b]_{k},
$$

for some $\mathrm{c} \in \mathbb{R}$.

\section{Main results obtained by the isochronous variational principle}

In this section, we intend to study Birkhoff equations, Noether identity and Noether theorem for Birkhoffian systems on time scales with nabla derivatives.

\subsection{Birkhoff equations with nabla derivatives}

We consider the following problem

$$
\bar{S}\left(a_{i}(\cdot)\right)=\int_{c}^{d}\left[R_{j}\left(s, a_{i}^{\rho}(s)\right) \cdot a_{j}^{\nabla}(s)-B\left(s, a_{i}^{\rho}(s)\right)\right] \nabla s \rightarrow \min ,
$$

for all $a_{i}(\cdot) \in C_{l d}^{1}$ with $a_{i}(c)=A_{i}, a_{i}(d)=B_{i}$, where $a_{i}^{\rho}(s)=\left(a_{i} \circ \rho\right)(s), a_{j}^{\nabla}(s)$ is the nabla derivatives, $B: \mathbb{R} \times \mathbb{R}^{2 n} \rightarrow \mathbb{R}, B \in C_{l d}^{1}$ is called Birkhoffian, $R_{j}: \mathbb{R} \times \mathbb{R}^{2 n} \rightarrow \mathbb{R}, R_{j} \in C_{l d}^{1}$ are called Birkhoff functions, $i, j=1,2, \cdots, 2 n$. We denote the partial derivatives of $R_{j}$ and $B$ with respect to their first variable by $\partial_{0} R_{j}$ and $\partial_{0} B$, and denote the partial derivatives of $R_{j}, B$ with respect to their $l+1-$ th variable by $\partial_{l} R_{j}, \partial_{l} B$, $l=1,2, \cdots, 2 n$.

The isochronous variational principle

$$
\delta \bar{S}=0
$$


with the exchange relationships

$$
\left(\delta a_{i}\right)^{\nabla}=\delta a_{i}^{\nabla}, \quad\left(\delta a_{i}\right)^{\rho}=\delta a_{i}^{\rho},
$$

and the boundary value conditions

$$
\left.\delta a_{i}\right|_{s=c}=\left.\delta a_{i}\right|_{s=d}=0,
$$

is called Pfaff-Birkhoff principle on time scales with nabla derivatives, where formula (3.3) can be proved as $\left(\delta a_{i}\right)^{\triangle}=\delta a_{i}^{\triangle},\left(\delta a_{i}\right)^{\sigma}=\delta a_{i}^{\sigma}$ in [29].

Expanding formula (3.2), we obtain

$$
\begin{aligned}
\delta \bar{S} & =\int_{c}^{d}\left[\delta R_{j}\left(s, a_{i}^{\rho}\right) \cdot a_{j}^{\nabla}+R_{j}\left(s, a_{i}^{\rho}\right) \cdot \delta a_{j}^{\nabla}-\delta B\left(s, a_{i}^{\rho}\right)\right] \nabla s \\
& =\int_{c}^{d}\left[\partial_{l} R_{j}\left(s, a_{i}^{\rho}\right) \delta a_{l}^{\rho} \cdot a_{j}^{\nabla}+R_{j}\left(s, a_{i}^{\rho}\right) \cdot \delta a_{j}^{\nabla}-\partial_{l} B\left(s, a_{i}^{\rho}\right) \delta a_{l}^{\rho}\right] \nabla s \\
& =\int_{c}^{d}\left\{\left[-\int_{c}^{s}\left(\partial_{l} R_{j}\left(\tau, a_{i}^{\rho}(\tau)\right) \cdot a_{j}^{\nabla}(\tau)\right) \nabla \tau+R_{l}+\int_{c}^{s} \partial_{l} B\left(\tau, a_{i}^{\rho}(\tau)\right) \nabla \tau\right]\left(\delta a_{l}\right) \nabla\right\} \nabla s \\
& =0 .
\end{aligned}
$$

Hence, from Lemma 2.3, we get

$$
\partial_{l} R_{j}\left(s, a_{i}^{\rho}\right) \cdot a_{j}^{\nabla}-\partial_{l} B\left(s, a_{i}^{\rho}\right)-R_{l}^{\nabla}=0 .
$$

Equations (3.4) are called Birkhoff equations on time scales with nabla derivatives.

\subsection{Noether theorem with nabla derivatives}

Firstly, we consider the infinitesimal transformations without transforming the time.

Definition 3.1. Under the following transformations

$$
\tilde{s}=s, \quad \tilde{a_{i}}(s)=a_{i}(s)+\varepsilon \xi_{i}\left(s, a_{j}\right)+o(\varepsilon),
$$

where $\varepsilon$ is an infinitesimal parameter, $\xi_{i}, i=1,2, \cdots, 2 n$ are called the infinitesimal generators of the transformations, formula (3.1) is said to be invariant if and only if

$$
\int_{s_{c}}^{s_{d}}\left[R_{j}\left(s, a_{i}^{\rho}\right) a_{j}^{\nabla}-B\left(s, a_{i}^{\rho}\right)\right] \nabla s=\int_{s_{c}}^{s_{d}}\left[R_{j}\left(s, \tilde{a}_{i}^{\rho}\right) \tilde{a}_{j}^{\nabla}-B\left(s, \tilde{a}_{i}^{\rho}\right)\right] \nabla s,
$$

holds for any $\left[s_{c}, s_{d}\right] \subseteq[c, d]$.

Definition 3.2. A quantity $I\left(s, a_{i}, a_{i}^{\rho}, a_{i}^{\nabla}\right)$ (resp. $\left.I\left(s, a_{i}, a_{i}^{\sigma}, a_{i}^{\triangle}\right)\right)$ is said to be a conserved quantity of the Birkhoffian system with nabla derivatives (resp. the Birkhoffian system with delta derivatives) if and only if $\frac{\nabla}{\nabla s} \mathrm{I}\left(\mathrm{s}, \mathrm{a}_{i}, \mathrm{a}_{i}^{\rho}, \mathrm{a}_{i}^{\nabla}\right)=0$ (resp. $\frac{\Delta}{\Delta s} \mathrm{I}\left(\mathrm{s}, \mathrm{a}_{i}, \mathrm{a}_{i}^{\sigma}, \mathrm{a}_{i}^{\triangle}\right)=0$ ) holds along the equations of motion for the system.

Theorem 3.3. Under the transformations (3.5), if formula (3.1) is invariant, then

$$
\partial_{l} R_{j}\left(s, a_{i}^{\rho}\right) \xi_{l}^{\rho} a_{j}^{\nabla}+R_{j}\left(s, a_{i}^{\rho}\right) \xi_{j}^{\nabla}-\partial_{l} B\left(s, a_{i}^{\rho}\right) \xi_{l}^{\rho}=0,
$$

where $\xi_{i}^{\rho}\left(s, a_{j}\right)=\xi_{i}\left(\rho(s), a_{j}(\rho(s))\right), \xi_{i}^{\nabla}\left(s, a_{j}\right)=\frac{\nabla}{\nabla s} \xi_{i}\left(s, a_{j}\right)$.

Proof. From Definition 3.1, we get

$$
R_{j}\left(s, a_{i}^{\rho}\right) a_{j}^{\nabla}-B\left(s, a_{i}^{\rho}\right)=R_{j}\left(s, a_{i}^{\rho}+\varepsilon \xi_{i}^{\rho}\right)\left(a_{j}^{\nabla}+\varepsilon \xi_{j}^{\nabla}\right)-B\left(s, a_{i}^{\rho}+\varepsilon \xi_{i}^{\rho}\right) .
$$

Differentiating both sides of formula (3.7) with respect to $\varepsilon$ and letting $\varepsilon=0$, we can get the intended result. 
Theorem 3.4. If formula (3.1) is invariant under Definition 3.1, then

$$
I\left(s, a_{i}, a_{i}^{\rho}, a_{i}^{\nabla}\right)=R_{j}\left(s, a_{i}^{\rho}\right) \xi_{j}\left(s, a_{i}\right),
$$

is a conserved quantity for the Birkhoffian system (3.4).

Proof. From (3.4) and formula (3.6), we have

$$
\begin{aligned}
\frac{\nabla}{\nabla s} \mathrm{I}\left(\mathrm{s}, \mathrm{a}_{i}, \mathrm{a}_{i}^{\rho}, \mathrm{a}_{i}^{\nabla}\right) & =\frac{\nabla}{\nabla s}\left[\mathrm{R}_{j}\left(s, \mathrm{a}_{i}^{\rho}\right) \xi_{j}\left(s, a_{i}\right)\right]=R_{j} \xi_{j}^{\nabla}+R_{j}^{\nabla} \xi_{j}^{\rho} \\
& =R_{j} \xi_{j}^{\nabla}+\left[\partial_{l} R_{i}\left(s, a_{j}^{\rho}\right) \cdot a_{i}^{\nabla}-\partial_{l} B\left(s, a_{j}^{\rho}\right)\right] \xi_{l}^{\rho}=0 .
\end{aligned}
$$

Secondly, we consider the general infinitesimal transformations:

$$
\tilde{s}=P\left(s, a_{j}, \varepsilon\right)=s+\varepsilon \xi_{0}\left(s, a_{j}\right)+o(\varepsilon), \quad \tilde{a_{i}}(\tilde{s})=Q_{i}\left(s, a_{j}, \varepsilon\right)=a_{i}(s)+\varepsilon \xi_{i}\left(s, a_{j}\right)+o(\varepsilon),
$$

where $\varepsilon$ is an infinitesimal parameter, $\xi_{0}:[c, d] \times \mathbb{R}^{2 n} \rightarrow \mathbb{R}$ and $\xi_{i}:[c, d] \times \mathbb{R}^{2 n} \rightarrow \mathbb{R}$ are the infinitesimal generators, and both of them are nabla differential functions.

Let $\mathrm{U}=\left\{\mathrm{a}_{\mathrm{i}} \mid \mathrm{a}_{\mathrm{i}}:[\mathrm{c}, \mathrm{d}] \rightarrow \mathbb{R}, \mathrm{a}_{\mathrm{i}} \in \mathrm{C}_{\mathrm{ld}}^{1}\right\} \subseteq \mathrm{C}_{\mathrm{ld}}^{1}$, suppose that the map $\mathrm{s} \in[\mathrm{c}, \mathrm{d}] \mapsto \alpha(\mathrm{s}):=\mathrm{P}\left(\mathrm{s}, \mathrm{a}_{\mathrm{j}}, \varepsilon\right) \in \mathbb{R}$ is an increasing $C_{l d}^{1}$ function and its image is a new time scale, whose backward jump operator and nabla operator are $\tilde{\rho}$ and $\tilde{\nabla}$, respectively. And we can easily get

$$
\tilde{\rho} \circ \alpha=\alpha \circ \rho .
$$

Definition 3.5. Under the transformations (3.8), formula (3.1) is said to be invariant if and only if

$$
\int_{s_{c}}^{s_{d}}\left[R_{j}\left(s, a_{i}^{\rho}\right) a_{j}^{\nabla}-B\left(s, a_{i}^{\rho}\right)\right] \nabla s=\int_{\tilde{s_{c}}}^{\tilde{s_{d}}}\left[R_{j}\left(\tilde{s}, \tilde{a_{i}} \tilde{\rho}(\tilde{s})\right) \tilde{a_{j}} \tilde{\nabla}(\tilde{s})-B\left(\tilde{s}, \tilde{a_{i}} \tilde{\rho}(\tilde{s})\right)\right] \tilde{\nabla} \tilde{s},
$$

holds for any $\left[s_{c}, s_{d}\right] \subseteq[c, d]$.

Theorem 3.6. Under the infinitesimal transformations (3.8), formula (3.1) is invariant if and only if

$$
\left(\partial_{0} R_{j} \cdot a_{j}^{\nabla}-\partial_{0} B\right) \xi_{0}+\left(\partial_{i} R_{j} \cdot a_{j}^{\nabla}-\partial_{i} B\right) \xi_{i}^{\rho}+R_{j} \xi_{j}^{\nabla}-B \xi_{0}^{\nabla}=0 .
$$

Proof. Since

$$
\begin{aligned}
\int_{s_{c}}^{s_{d}} & \left.R_{j}\left(s, a_{i}^{\rho}\right) a_{j}^{\nabla}-B\left(s, a_{i}^{\rho}\right)\right] \nabla s \\
& \left.=\int_{\alpha\left(s_{c}\right)}^{\alpha\left(s_{d}\right)}\left[R_{j}\left(\tilde{s}, \tilde{a_{i}} \tilde{\rho}(\tilde{s})\right) \tilde{a_{j}} \tilde{\nabla}(\tilde{(s})\right)-B\left(\tilde{s}, \tilde{a_{i}} \tilde{\rho}(\tilde{s})\right)\right] \tilde{\nabla} \tilde{s} \\
& =\int_{s_{c}}^{s_{d}}\left[R_{j}\left(\alpha(s),\left(\tilde{a_{i}} \circ \tilde{\rho} \circ \alpha\right)(s)\right) \tilde{a_{j}} \tilde{\nabla}(\alpha(s))-B\left(\alpha(s),\left(\tilde{a_{i}} \circ \tilde{\rho} \circ \alpha\right)(s)\right)\right] \alpha \nabla(s) \nabla s \\
& =\int_{s_{c}}^{s_{d}}\left[R_{j}\left(\alpha(s),\left(\tilde{a_{i}} \circ \alpha \circ \rho\right)(s)\right) \frac{\left(\tilde{a_{j}} \circ \alpha\right)^{\nabla}(s)}{\alpha \nabla(s)}-B\left(\alpha(s),\left(\tilde{a_{i}} \circ \alpha \circ \rho\right)(s)\right)\right] \alpha^{\nabla}(s) \nabla s \\
& =\int_{s_{c}}^{s_{d}}\left[R_{j}\left(P, Q_{i}^{\rho}\right) \frac{Q_{j}^{\nabla}}{P \nabla}-B\left(P, Q_{i}^{\rho}\right)\right] P^{\nabla} \nabla s,
\end{aligned}
$$

holds for any $\left[s_{c}, s_{d}\right] \subseteq[c, d]$, we have

$$
R_{j}\left(s, a_{i}^{\rho}\right) a_{j}^{\nabla}-B\left(s, a_{i}^{\rho}\right)=\left[R_{j}\left(P, Q_{i}^{\rho}\right) \frac{Q_{j}^{\nabla}}{P \nabla}-B\left(P, Q_{i}^{\rho}\right)\right] P^{\nabla} .
$$

Then differentiating both sides of formula (3.10) with respect to $\varepsilon$ and letting $\varepsilon=0$, we can get the intended result. 
Formula (3.9) is called Noether identity for Birkhoffian systems on time scales with nabla derivatives.

Theorem 3.7. If formula (3.1) is invariant under Definition 3.5, then

$$
I\left(s, a_{j}, a_{j}^{\rho}, a_{j}^{\nabla}\right)=R_{i}\left(s, a_{j}^{\rho}\right) \xi_{i}\left(s, a_{j}\right)+\left[v(s)\left(\partial_{0} R_{j} \cdot a_{j}^{\nabla}-\partial_{0} B\right)-B\right] \xi_{0},
$$

is a conserved quantity for the Birkhoffian system on time scales with nabla derivatives.

Proof. The idea of this proof is to transform the invariance of formula (3.1) under Definition 3.5 to the invariance of formula (3.1) under Definition 3.1, and then make use of Theorem 3.4.

We denote

$$
\begin{aligned}
\bar{S}\left(a_{i}(\cdot)\right) & =\int_{s_{c}}^{s_{d}}\left[R_{j}\left(s, a_{i}^{\rho}\right) a_{j}^{\nabla}-B\left(s, a_{i}^{\rho}\right)\right] \nabla s \doteq \int_{s_{c}}^{s_{d}} G\left(s, a_{i}^{\rho}, a_{i}^{\nabla}\right) \nabla s . \\
\bar{S}\left(\theta(\cdot), a_{i}(\cdot)\right) & =\int_{s_{c}}^{s_{d}}\left[R_{j}\left(\theta^{\rho}+v(s) \theta^{\nabla}, a_{i}^{\rho}\right) \frac{a_{j}^{\nabla}}{\theta^{\nabla}}-B\left(\theta^{\rho}+v(s) \theta^{\nabla}, a_{i}^{\rho}\right)\right] \theta^{\nabla} \nabla s \\
& \doteq \int_{s_{c}}^{s_{d}} \bar{G}\left(s, \theta^{\rho}, a_{i}^{\rho}, \theta^{\nabla}, a_{i}^{\nabla}\right) \nabla s .
\end{aligned}
$$

When $\theta(s)=s$, we have

$$
\int_{s_{c}}^{s_{d}}\left[R_{j}\left(s, a_{i}^{\rho}\right) a_{j}^{\nabla}-B\left(s, a_{i}^{\rho}\right)\right] \nabla s=\int_{s_{c}}^{s_{d}}\left[R_{j}\left(\theta^{\rho}+v(s) \theta^{\nabla}, a_{i}^{\rho}\right) \frac{a_{j}^{\nabla}}{\theta^{\nabla}}-B\left(\theta^{\rho}+v(s) \theta^{\nabla}, a_{i}^{\rho}\right)\right] \theta^{\nabla} \nabla s .
$$

That is,

$$
\overline{\mathrm{S}}\left(\mathrm{a}_{\mathfrak{i}}(\cdot)\right)=\overline{\overline{\mathrm{S}}}\left(\theta(\cdot), \mathrm{a}_{\mathfrak{i}}(\cdot)\right)
$$

Hence, when $\theta(s)=s$, we have

$$
\begin{aligned}
\overline{\bar{S}}\left(\theta(\cdot), \tilde{a}_{i}(\cdot)\right) & =\bar{S}\left(a_{i}(\cdot)\right)=\int_{s_{c}}^{s_{d}}\left[R_{j}\left(s, a_{i}^{\rho}\right) a_{j}^{\nabla}-B\left(s, a_{i}^{\rho}\right)\right] \nabla s \\
& \left.=\int_{\alpha\left(s_{c}\right)}^{\alpha\left(s_{d}\right)}\left[R_{j}\left(\tilde{s}, \tilde{a_{i}} \tilde{\rho}(\tilde{s})\right) \tilde{a_{j}} \tilde{\nabla}(\tilde{(} s)\right)-B\left(\tilde{s}, \tilde{a_{i}} \tilde{\rho}(\tilde{s})\right)\right] \tilde{\nabla} \tilde{s} \\
& =\int_{s_{c}}^{s_{d}}\left[R_{j}\left(\alpha(s),\left(\tilde{a_{i}} \circ \tilde{\rho} \circ \alpha\right)(s)\right) \tilde{a_{j}} \tilde{\nabla}(\alpha(s))-B\left(\alpha(s),\left(\tilde{a_{i}} \circ \tilde{\rho} \circ \alpha\right)(s)\right)\right] \alpha^{\nabla}(s) \nabla s \\
& =\int_{s_{c}}^{s_{d}}\left[R_{j}\left(\alpha(s),\left(\tilde{a_{i}} \circ \alpha \circ \rho\right)(s)\right) \frac{\left(\tilde{a_{j}} \circ \alpha\right)^{\nabla}(s)}{\alpha \nabla(s)}-B\left(\alpha(s),\left(\tilde{a_{i}} \circ \alpha \circ \rho\right)(s)\right)\right] \alpha^{\nabla}(s) \nabla s \\
& =\int_{s_{c}}^{s_{d}}\left[R_{j}\left(\alpha^{\rho}+v(s) \alpha^{\nabla},\left(\tilde{a_{i}} \circ \alpha\right)^{\rho}(s)\right) \frac{\left(\tilde{a_{j}} \circ \alpha\right)^{\nabla}}{\alpha \nabla}-B\left(\alpha^{\rho}+v(s) \alpha \nabla,\left(\tilde{a_{i}} \circ \alpha\right)^{\rho}(s)\right)\right] \alpha^{\nabla} \nabla s \\
& \doteq \int_{s_{c}}^{s_{d}} \bar{G}\left(s, \alpha^{\rho},\left(\tilde{a_{i}} \circ \alpha\right)^{\rho}, \alpha \nabla,\left(\tilde{a_{i}} \circ \alpha\right)^{\nabla}\right) \nabla s=\overline{\bar{S}}\left(\alpha(\cdot),\left(\tilde{a_{i}} \circ \alpha\right)(\cdot)\right) .
\end{aligned}
$$

For $\theta(s)=s$, we have

$$
\left(\alpha(s),\left(\tilde{a_{i}} \circ \alpha\right)(s)\right)=\left(P\left(s, a_{j}, \varepsilon\right), Q_{i}\left(s, a_{j}, \varepsilon\right)\right)=\left(P\left(\theta(s), a_{j}, \varepsilon\right), Q_{i}\left(\theta(s), a_{j}, \varepsilon\right)\right) .
$$

That is, $\overline{\bar{S}}$ is invariant on $\overline{\bar{U}}=\left\{\left(\theta, a_{i}\right) \mid \theta(s)=s, a_{i} \in U\right\}$ under the following transformations

$$
\left(\tilde{\theta}, \tilde{a_{i}}\right)=\left(P\left(\theta, a_{j}, \varepsilon\right), Q_{i}\left(\theta, a_{j}, \varepsilon\right)\right),
$$

in the sense of Definition 3.1. Therefore, when $\theta(s)=s$, we get from Theorem 3.4 that

$$
I=\frac{\partial \bar{G}\left(s, \theta^{\rho}, a_{i}^{\rho}, \theta^{\nabla}, a_{i}^{\nabla}\right)}{\partial \theta^{\nabla}} \xi_{0}+\frac{\partial \bar{G}\left(s, \theta^{\rho}, a_{i}^{\rho}, \theta^{\nabla}, a_{i}^{\nabla}\right)}{\partial a_{j}^{\nabla}} \xi_{j},
$$


where

$$
\begin{aligned}
& \frac{\partial \bar{G}\left(s, \theta^{\rho}, a_{i}^{\rho}, \theta^{\nabla}, a_{i}^{\nabla}\right)}{\partial a_{j}^{\nabla}}= \\
& \begin{aligned}
\frac{\partial \bar{G}\left(s, \theta^{\rho}, a_{i}^{\rho}, \theta^{\nabla}, a_{i}^{\nabla}\right)}{\partial \theta^{\nabla}}= & v\left(\theta^{\rho}+v(s) \partial_{0} R_{j}\left(\theta^{\rho}+v(s) \theta^{\nabla}, a_{i}^{\rho}\right)=R_{j}\left(s, a_{i}^{\rho}\right), a_{j}^{\nabla}\right. \\
& -v(s) \partial_{0} B\left(\theta^{\rho}+v(s) \theta^{\nabla}, a_{i}^{\rho}\right) \cdot \theta^{\nabla}-B\left(\theta^{\rho}+v(s) \theta^{\nabla}, a_{i}^{\rho}\right) \\
= & v(s)\left[\partial_{0} R_{j}\left(s, a_{i}^{\rho}\right) \cdot a_{j}^{\nabla}-\partial_{0} B\left(s, a_{i}^{\rho}\right)\right]-B\left(s, a_{i}^{\rho}\right) .
\end{aligned}
\end{aligned}
$$

The intended result can be obtained by substituting formula (3.12) and formula (3.13) into formula (3.11).

\subsection{Special cases}

In this section, we discuss some special cases such as the continuous Birkhoffian system, the discrete Birkhoffian system, the Hamiltonian system on time scales with nabla derivatives, as well as the Lagrangian system on time scales with nabla derivatives.

Corollary 3.8. If $\mathbb{T}=\mathbb{R}$, we have

$$
\rho(s)=s, \quad v(s)=0, \quad f^{\nabla}(s)=\dot{f}(s) .
$$

In this case, (3.4) reduces to the classical Birkhoff equations

$$
\left(\frac{\partial R_{j}}{\partial a_{i}}-\frac{\partial R_{i}}{\partial a_{j}}\right) \dot{a}_{j}-\frac{\partial B\left(s, a_{j}\right)}{\partial a_{i}}-\frac{\partial R_{i}\left(s, a_{j}\right)}{\partial s}=0 .
$$

Formula (3.9) gives the classical Noether identity for the Birkhoffian system (3.14)

$$
\left(\frac{\partial R_{j}}{\partial s}-\frac{\partial B}{\partial s}\right) \xi_{0}+\left(\frac{\partial R_{j}}{\partial a_{i}} \dot{a}_{j}-\frac{\partial B}{\partial a_{i}}\right) \xi_{i}+R_{j} \dot{\xi}_{j}-B \dot{\xi}_{0}=0 .
$$

And Theorem 3.7 gives the classical conserved quantity for the Birkhoffian system (3.14)

$$
I=R_{i}\left(s, a_{j}\right) \xi_{i}\left(s, a_{j}\right)-B \xi_{0}=\text { const. . }
$$

The results in Corollary 3.8 coincide with the results in [37].

Corollary 3.9. Consider the following transformations:

$$
a_{i}^{\rho}=\left\{\begin{array}{cc}
q_{i}^{\rho}, & i=1,2, \cdots, n \\
p_{i-n}, & i=n+1, n+2, \cdots, 2 n
\end{array}, \quad R_{i}=\left\{\begin{array}{cc}
p_{i}, & i=1,2, \cdots, n \\
0, & i=n+1, n+2, \cdots, 2 n
\end{array}, \quad B=H,\right.\right.
$$

where $\mathrm{q}_{\mathrm{m}}$ denotes the generalized coordinate, $\mathrm{p}_{\mathrm{m}}$ denotes the generalized momentum, $\mathrm{m}=1,2, \cdots, 2 \mathrm{n}, \mathrm{H}$ is the Hamiltonian. From formula (3.15), we can get the following Hamilton action with nabla derivatives

$$
\overline{\mathrm{S}}_{\mathrm{H}}=\int_{\mathrm{c}}^{\mathrm{d}}\left(\mathrm{p}_{\mathrm{m}} \mathrm{q}_{\mathrm{m}}^{\nabla}-\mathrm{H}\right) \nabla \mathrm{s}, \quad \mathrm{m}=1,2, \cdots, \mathrm{n} .
$$

Then (3.4) reduces to the Hamilton canonical equations on time scales with nabla derivatives

$$
q_{x}^{\nabla}=\frac{\partial H\left(s, q_{m}^{\rho}, p_{m}\right)}{\partial p_{x}}, \quad p_{x}^{\nabla}=-\frac{\partial H\left(s, q_{m}^{\rho}, p_{m}\right)}{\partial q_{x}^{\rho}}, \quad x=1,2, \cdots, n .
$$

Formula (3.9) gives the Noether identity for the Hamiltonian system (3.16)

$$
p_{m} \xi_{m}^{\nabla}-\frac{\partial H\left(s, q_{m}^{\rho}, p_{m}\right)}{\partial s} \xi_{0}-\frac{\partial H}{\partial q_{m}^{\rho}} \xi_{m}^{\rho}-H \xi_{0}^{\nabla}=0 .
$$

And Theorem 3.7 gives the conserved quantity for the Hamiltonian system (3.16)

$$
\mathrm{I}_{\mathrm{H}}=\mathrm{p}_{\mathrm{m}} \xi_{\mathrm{m}}-\left[v(\mathrm{~s}) \frac{\partial \mathrm{H}}{\partial \mathrm{s}}+\mathrm{H}\right] \xi_{0}=\text { const. . }
$$


Corollary 3.10. Consider

$$
p_{\chi}=\frac{\partial L\left(s, q_{m}^{\rho}, q_{m}^{\nabla}\right)}{\partial q_{\chi}^{\nabla}}, \quad H=p_{\chi} q_{\chi}^{\nabla}-L,
$$

where $\mathrm{L}$ is the Lagrangian. Then Euler-Lagrange equations, Noether identity and conserved quantity for Lagrangian systems on time scales with nabla derivatives can be achieved as follows

$$
\begin{gathered}
\frac{\nabla}{\nabla s} \frac{\partial \mathrm{L}\left(\mathrm{s}, \mathrm{q}_{\mathrm{m}}^{\rho}, \mathrm{q}_{\mathrm{m}}^{\nabla}\right)}{\partial \mathrm{q}_{\chi}^{\nabla}}=\frac{\partial \mathrm{L}\left(\mathrm{s}, \mathrm{q}_{\mathrm{m}}^{\rho}, \mathrm{q}_{\mathrm{m}}^{\nabla}\right)}{\partial \mathrm{q}_{\chi}^{\rho}}, \\
\frac{\partial \mathrm{L}\left(\mathrm{s}, \mathrm{q}_{\mathrm{m}}^{\rho}, \mathrm{q}_{\mathrm{m}}^{\nabla}\right)}{\partial \mathrm{s}} \xi_{0}+\frac{\partial \mathrm{L}}{\partial \mathrm{q}_{\chi}^{\rho}} \xi_{\chi}^{\rho}+\frac{\partial \mathrm{L}}{\partial \mathrm{q}_{\chi}^{\nabla}} \xi_{\chi}^{\nabla}+\mathrm{L}_{0}^{\nabla}-\frac{\partial \mathrm{L}}{\partial \mathrm{q}_{\chi}^{\nabla}} \mathrm{q}_{\chi}^{\nabla} \xi_{0}^{\nabla}=0, \\
\mathrm{I}_{\mathrm{L}}=\frac{\partial \mathrm{L}\left(\mathrm{s}, \mathrm{q}_{\mathrm{m}}^{\rho}, \mathrm{q}_{\mathrm{m}}^{\nabla}\right)}{\partial \mathrm{q}_{\chi}^{\nabla}} \xi_{\chi}^{\nabla}+\left[\mathrm{L}-\frac{\partial \mathrm{L}}{\partial \mathrm{q}_{\mathrm{m}}^{\nabla}} \mathrm{q}_{\mathrm{m}}^{\nabla}+v(\mathrm{~s}) \frac{\partial \mathrm{L}}{\partial \mathrm{s}}\right] \xi_{0}=\text { const. . }
\end{gathered}
$$

The results in Corollary 3.10 coincide with the results in [30,55].

In the remainder of this section, we consider the discrete Birkhoffian system in detail. In this section, we restrict ourselves to the time scale $\mathbb{T}=\{c, c+1, \cdots, d\}$, where $c, d \in \mathbb{T}$. And we assume that $\mathbb{T}$ has enough points to ensure the following calculations. It is obvious that $[c, d]_{\kappa}=[\sigma(c), d]$. Since $\mathbb{T}=\mathbb{Z}$, we have

$$
\rho(s)=s-1, \quad v(s)=1, \quad f^{\nabla}(s)=f(s)-f^{\rho}(s) \doteq \nabla_{n} f(s) .
$$

Then formula (3.1) gives the following discrete Pfaff action

$$
\bar{S}_{D}=\sum_{s=c+1}^{d}\left[R_{j}\left(s, a_{i}^{\rho}\right) \nabla_{n} a_{j}-B\left(s, a_{i}^{\rho}\right)\right] .
$$

The isochronous variational principle

$$
\delta \bar{S}_{\mathrm{D}}=0,
$$

with the exchange relationships

$$
\nabla_{n}\left(\delta a_{i}\right)=\delta\left(\nabla_{n} a_{i}\right), \quad \delta a_{i}^{\rho}=\left(\delta a_{i}\right)^{\rho},
$$

and boundary value conditions

$$
\left.\delta a_{i}\right|_{s=c}=\left.\delta a_{i}\right|_{s=d}=0,
$$

is called discrete Pfaff-Birkhoff principle.

Using the discrete Pfaff-Birkhoff principle, we can derive

$$
\frac{\partial R_{j}}{\partial a_{i}^{\rho}} \cdot \nabla_{n} a_{j}-\nabla_{n} R_{i}-\frac{\partial B}{\partial a_{i}^{\rho}}=0, \quad s \in\{c+2, c+3, \cdots, d\} .
$$

Equations (3.17) are the discrete Birkhoff equations.

Formula (3.9) gives the discrete Noether identity for the discrete Birkhoffian system

$$
\left(\frac{\partial R_{j}}{\partial s} \nabla_{n} a_{j}-\frac{\partial B}{\partial s}\right) \xi_{0}+\left(\frac{\partial R_{j}}{\partial a_{i}^{\rho}} \nabla_{n} a_{j}-\frac{\partial B}{\partial a_{i}^{\rho}}\right) \xi_{i}^{\rho}+R_{j} \cdot \nabla_{n} \xi_{j}-B \cdot \nabla_{n} \xi_{0}=0 .
$$

And Theorem 3.7 gives the discrete conserved quantity for the discrete Birkhoffian system

$$
I\left(s, a_{j}, a_{j}^{\rho}, \nabla_{n} a_{j}\right)=R_{i}\left(s, a_{j}^{\rho}\right) \xi_{i}\left(s, a_{j}\right)+\left[\left(\frac{\partial R_{j}}{\partial s} \cdot \nabla_{n} a_{j}-\frac{\partial B}{\partial s}\right)-B\right] \xi_{0}=\text { const. . }
$$

Remark 3.11. It follows from the results of the discrete Birkhoffian system that time scale is an important tool to study the discrete problems. 


\section{Main results obtained by the duality principle}

In this section, we use the duality principle to get the Birkhoff equations, the Noether identity and the conserved quantity for the Birkhoffian system on time scales with nabla derivatives.

\subsection{Results with delta derivatives}

Some results for the Birkhoffian system on time scales with delta derivatives are reviewed, see [66].

We consider the following problem

$$
S\left(a_{i}(\cdot)\right)=\int_{a}^{b}\left[R_{j}\left(t, a_{i}^{\sigma}(t)\right) \cdot a_{j}^{\triangle}(t)-B\left(t, a_{i}^{\sigma}(t)\right)\right] \triangle t \rightarrow \min ,
$$

under the given boundary conditions $a_{i}(a)=\alpha_{i}, a_{i}(b)=\beta_{i}$, where $a_{i}^{\sigma}(t)=\left(a_{i} \circ \sigma\right)(t), a_{j}^{\triangle}(t)=\frac{\Delta}{\Delta t} a_{j}(t)$, $t \in \mathbb{T}, a_{i}(\cdot) \in C_{r d}^{1}$, the Birkhoff's functions $R_{j}: \mathbb{R} \times \mathbb{R}^{2 n} \rightarrow \mathbb{R}$, and the Birkhoffian $B: \mathbb{R} \times \mathbb{R}^{2 n} \rightarrow \mathbb{R}$ are all $\mathrm{C}_{\text {rd }}^{1}$ functions, $i, j=1,2, \cdots, 2 \mathrm{n}$.

Theorem 4.1. If problem (4.1) has a minimizer $\mathrm{a}_{\mathrm{i} 0}(\cdot)$, then we have the following Birkhoff equations on time scales for all $\mathrm{t} \in[\mathrm{a}, \mathrm{b}]^{\mathrm{K}}$ :

$$
\partial_{l} R_{j}\left(t, a_{i 0}^{\sigma}(t)\right) \cdot a_{j}^{\triangle}(t)-\partial_{l} B\left(t, a_{i 0}^{\sigma}(t)\right)-R_{l}^{\triangle}\left(t, a_{i 0}^{\sigma}(t)\right)=0, \quad i, j, l=1,2, \cdots, 2 n .
$$

Equations (4.2) are the Birkhoff equations on time scales with delta derivatives.

Theorem 4.2. If formula (4.1) is invariant under the transformations

$$
\tilde{\mathrm{t}}=Y\left(t, a_{i}, \varepsilon\right)=t+\varepsilon \zeta_{0}\left(t, a_{i}\right)+o(\varepsilon), \quad \tilde{a_{j}}(\tilde{t})=Z_{j}\left(t, a_{i}, \varepsilon\right)=a_{j}(t)+\varepsilon \zeta_{j}\left(t, a_{i}\right)+o(\varepsilon),
$$

then we have

$$
\begin{aligned}
& {\left[\partial_{0} R_{j}\left(t, a_{i}^{\sigma}(t)\right) \cdot a_{j}^{\triangle}(t)-\partial_{0} B\left(t, a_{i}^{\sigma}(t)\right)\right] \cdot \zeta_{0}\left(t, a_{i}(t)\right)+R_{j}\left(t, a_{i}^{\sigma}(t)\right) \zeta_{j}^{\triangle}\left(t, a_{i}(t)\right)} \\
& \quad+\left[\partial_{l} R_{j}\left(t, a_{i}^{\sigma}(t)\right) \cdot a_{j}^{\triangle}(t)-\partial_{l} B\left(t, a_{i}^{\sigma}(t)\right)\right] \cdot \zeta_{l}^{\sigma}\left(t, a_{i}(t)\right)-B\left(t, a_{i}^{\sigma}(t)\right) \zeta_{0}^{\triangle}\left(t, a_{i}(t)\right)=0 .
\end{aligned}
$$

Formula (4.4) is the Noether identity for the Birkhoffian system on time scales with delta derivatives.

Theorem 4.3. If formula (4.1) is invariant under the transformations (4.3), then

$$
\begin{aligned}
I & =R_{j}\left(t, a_{i}^{\sigma}(t)\right) \cdot \zeta_{j}\left(t, a_{i}(t)\right)-\left\{\mu(t)\left[\partial_{0} R_{j}\left(t, a_{i}^{\sigma}(t)\right) \cdot a_{j}^{\triangle}(t)-\partial_{0} B\left(t, a_{i}^{\sigma}(t)\right)\right]+B\left(t, a_{i}^{\sigma}(t)\right)\right\} \cdot \zeta_{0}\left(t, a_{i}(t)\right) \\
& =\text { const. . }
\end{aligned}
$$

Theorem 4.3 is the Noether theorem for the Birkhoffian system on time scales with delta derivatives.

\subsection{Results with nabla derivatives}

In this section, we consider the problem (3.1) using the duality principle.

Definition 4.4. Given the Birkhoff's functions $R_{j}: \mathbb{T} \times \mathbb{R}^{2 n} \rightarrow \mathbb{R}$ and the Birkhoffian $B: \mathbb{T} \times \mathbb{R}^{2 n} \rightarrow \mathbb{R}$, we define the dual $R_{j}^{*}: \mathbb{T}^{*} \times \mathbb{R}^{2 n} \rightarrow \mathbb{R}$ and $B^{*}: \mathbb{T}^{*} \times \mathbb{R}^{2 n} \rightarrow \mathbb{R}$ by $R_{j}^{*}\left(x, y_{i}\right)=-R_{j}\left(-x, y_{i}\right)$ and $B^{*}\left(x, y_{i}\right)=$ $B\left(-x, y_{i}\right), i, j=1,2, \cdots, 2 n$.

Remark 4.5. It is easy to check that

$$
\begin{aligned}
& \partial_{0} R_{j}^{*}\left(x, y_{i}\right)=\partial_{0} R_{j}\left(-x, y_{i}\right), \\
& \partial_{l} R_{j}^{*}\left(x, y_{i}\right)=-\partial_{l} R_{j}\left(-x, y_{i}\right), \\
& \partial_{0} B^{*}\left(x, y_{i}\right)=-\partial_{0} B\left(-x, y_{i}\right),
\end{aligned}
$$

and

$$
\partial_{l} B^{*}\left(x, y_{i}\right)=\partial_{l} B\left(-x, y_{i}\right), \quad i, j, l=1,2, \cdots, 2 n .
$$


Proposition 4.6. Given the Birkhoff's functions $\mathrm{R}_{\mathbf{j}}: \mathbb{T} \times \mathbb{R}^{2 \mathrm{n}} \rightarrow \mathbb{R}$ and the Birkhoffian $\mathrm{B}: \mathbb{T} \times \mathbb{R}^{2 \mathrm{n}} \rightarrow \mathbb{R}$, then for all $\mathrm{a}_{\mathrm{i}} \in \mathrm{C}_{\mathrm{ld}}^{1}([\mathrm{c}, \mathrm{d}])$, we have

$$
\int_{c}^{d}\left[R_{j}\left(s, a_{i}^{\rho}(s)\right) \cdot a_{j}^{\nabla}(s)-B\left(s, a_{i}^{\rho}(s)\right)\right] \nabla s=\int_{-d}^{-c}\left[R_{j}^{*}\left(t,\left(a_{i}^{*}\right)^{\hat{\sigma}}(t)\right) \cdot\left(a_{j}^{*}\right)^{\hat{\Delta}}(t)-B^{*}\left(t,\left(a_{i}^{*}\right)^{\hat{\sigma}}(t)\right)\right] \hat{\Delta} t .
$$

Proof. From Proposition 2.2 and Definition 4.4, we have

$$
\begin{aligned}
R_{j}^{*}\left(t,\left(a_{i}^{*}\right)^{\hat{\sigma}}(t)\right) \cdot\left(a_{j}^{*}\right)^{\hat{\Delta}}(t)-B^{*}\left(t,\left(a_{i}^{*}\right)^{\hat{\sigma}}(t)\right) & =-R_{j}^{*}\left(t, a_{i}^{*}(-\rho(-t))\right) \cdot a_{j}^{\nabla}(-t)-B^{*}\left(t, a_{i}^{*}(-\rho(-t))\right) \\
& =R_{j}\left(-t, a_{i}^{\rho}(-t)\right) \cdot a_{j}^{\nabla}(-t)-B\left(-t, a_{i}^{\rho}(-t)\right) .
\end{aligned}
$$

Hence, we can get the intended result using Proposition 2.2.

Theorem 4.7. If problem (3.1) has a minimizer $\overline{\mathrm{a}}_{\mathfrak{i} 0}$, then we have the following Birkhoff equations on time scales for all $\mathrm{s} \in[\mathrm{c}, \mathrm{d}]_{\mathrm{K}}$ :

$$
\partial_{l} R_{j}\left(s, \bar{a}_{i 0}^{\rho}(s)\right) \cdot a_{j}^{\nabla}(s)-\partial_{l} B\left(s, \bar{a}_{i 0}^{\rho}(s)\right)-R_{l}^{\nabla}\left(s, \bar{a}_{i 0}^{\rho}(s)\right)=0, \quad i, j, l=1,2, \cdots, 2 n .
$$

Proof. Since $\overline{\mathfrak{a}}_{\mathfrak{i} 0}$ is a minimizer of problem (3.1), then it follows from Proposition 4.6 that $\overline{\mathrm{a}}_{\mathfrak{i} 0}^{*}$ is a minimizer for the following variational problem

$$
\bar{S}^{*}\left(g_{i}\right)=\int_{-d}^{-c}\left[R_{j}^{*}\left(t, g_{i}^{\hat{o}}(t)\right) \cdot\left(a_{j}^{*}\right)^{\hat{\Delta}}(t)-B^{*}\left(t, g_{i}^{\hat{\sigma}}(t)\right)\right] \hat{\triangle} t, \quad g_{i}(-c)=A_{i}, \quad g_{i}(-d)=B_{i},
$$

where $g_{i} \in C_{r d}^{1}$. Therefore, we can apply Theorem 4.1 to get the Birkhoff equations for all $t \in[-d,-c]^{k}$ as follows:

$$
\partial_{l} R_{j}^{*}\left(t,\left(\bar{a}_{i 0}^{*}\right)^{\hat{\sigma}}(t)\right) \cdot\left(a_{j}^{*}\right)^{\hat{\Delta}}(t)-\partial_{l} B^{*}\left(t,\left(\bar{a}_{i 0}^{*}\right)^{\hat{\sigma}}(t)\right)-\left(R_{l}^{*}\right)^{\hat{\Delta}}\left(t,\left(\bar{a}_{i 0}^{*}\right)^{\hat{\sigma}}(t)\right)=0 .
$$

The following work is to rewrite formula (4.5).

Since

$$
R_{\mathfrak{j}}^{*}\left(t,\left(\bar{a}_{\mathfrak{i} 0}^{*}\right)^{\hat{\sigma}}(t)\right)=-R_{\mathfrak{j}}\left(-t, \bar{a}_{\mathfrak{i} 0}^{\rho}(-t)\right), \quad B^{*}\left(t,\left(\bar{a}_{\mathfrak{i} 0}^{*}\right)^{\hat{\sigma}}(t)\right)=B\left(-t, \bar{a}_{\mathfrak{i} 0}^{\rho}(-t)\right),
$$

from Proposition 2.2 and Remark 4.5, we can obtain

$$
\begin{aligned}
& \partial_{l} R_{j}^{*}\left(t,\left(\bar{a}_{i 0}^{*}\right){ }^{\hat{\sigma}}(t)\right)=-\partial_{l} R_{j}\left(-t, \bar{a}_{i 0}^{\rho}(-t)\right), \quad \partial_{l} B^{*}\left(t,\left(\bar{a}_{i 0}^{*}\right) \hat{\sigma}(t)\right)=\partial_{l} B\left(-t, \bar{a}_{i 0}^{\rho}(-t)\right), \\
& \left(R_{l}^{*}\right)^{\hat{\Delta}}\left(t,\left(\bar{a}_{i 0}^{*}\right)^{\hat{\sigma}}(t)\right)=R_{l}^{\nabla}\left(-t, \bar{a}_{i 0}^{\rho}(-t)\right), \quad\left(a_{j}^{*}\right)^{\hat{\Delta}}(t)=-a_{j}^{\nabla}(-t) .
\end{aligned}
$$

Substituting formula (4.7) into formula (4.5), we have

$$
\partial_{l} R_{j}\left(-t, \bar{a}_{i 0}^{\rho}(-t)\right) \cdot a_{j}^{\nabla}(-t)-\partial_{l} B\left(-t, \bar{a}_{i 0}^{\rho}(-t)\right)-R_{l}^{\nabla}\left(-t, \bar{a}_{\mathfrak{i} 0}^{\rho}(-t)\right)=0 .
$$

Since $t \in[-d,-c]^{k}$, let $s=-t \in[c, d]_{k}$, we can get

$$
\partial_{l} R_{j}\left(s, \bar{a}_{i 0}^{\rho}(s)\right) \cdot a_{j}^{\nabla}(s)-\partial_{l} B\left(s, \bar{a}_{i 0}^{\rho}(s)\right)-R_{l}^{\nabla}\left(s, \bar{a}_{i 0}^{\rho}(s)\right)=0 .
$$

Theorem 4.8. If formula (3.1) is invariant under the infinitesimal transformations (3.8), then we have

$$
\begin{gathered}
{\left[\partial_{0} R_{j}\left(s, \bar{a}_{i 0}^{\rho}(s)\right) \cdot a_{j}^{\nabla}(s)-\partial_{0} B\left(s, \bar{a}_{i 0}^{\rho}(s)\right)\right] \cdot \xi_{0}\left(s, \bar{a}_{i 0}(s)\right)+\left[\partial_{l} R_{j}\left(s, \bar{a}_{i 0}^{\rho}(s)\right) \cdot a_{j}^{\nabla}(s)-\partial_{l} B\left(s, \bar{a}_{i 0}^{\rho}(s)\right)\right]} \\
\times \xi_{l}^{\rho}\left(s, \bar{a}_{i 0}(s)\right)+R_{j}\left(s, \bar{a}_{i 0}^{\rho}(s)\right) \cdot \xi_{j}^{\nabla}\left(s, \bar{a}_{i 0}(s)\right)-B\left(s, \bar{a}_{i 0}^{\rho}(s)\right) \cdot \xi_{0}^{\nabla}\left(s, \bar{a}_{i 0}(s)\right)=0,
\end{gathered}
$$

for all $\mathrm{s} \in[\mathrm{c}, \mathrm{d}]_{\mathrm{K}}$. 
Proof. If $\overline{\mathrm{S}}$ is invariant under the infinitesimal transformations (3.8) on $\mathrm{U}$, then $\overline{\mathrm{S}}^{*}$ is invariant on $\overline{\mathrm{U}}=$ $\left\{g_{i} \mid g_{i}:[-d,-c] \rightarrow \mathbb{R}, g_{i} \in C_{r d}^{1}\right\}$ under the infinitesimal transformations

$$
\tilde{\mathrm{t}}=\mathrm{t}-\varepsilon \xi_{0}^{*}\left(\mathrm{t}, \mathrm{g}_{\mathrm{i}}\right)+\mathrm{o}(\varepsilon), \quad \tilde{g_{j}}(\tilde{\mathrm{t}})=\mathrm{g}_{j}(\mathrm{t})+\varepsilon \xi_{j}^{*}\left(t, g_{i}\right)+o(\varepsilon),
$$

where $\xi_{0}^{*}(x, y)=\xi_{0}(-x, y), \xi_{j}^{*}(x, y)=\xi_{j}(-x, y)$. Hence, from Theorem 4.2 , for all $t \in[-d,-c]^{k}$, we get

$$
\begin{aligned}
& {\left[\partial_{0} R_{j}^{*}\left(t,\left(\bar{a}_{i 0}^{*}\right)^{\hat{\sigma}}(t)\right) \cdot\left(a_{j}^{*}\right)^{\hat{\Delta}}(t)-\partial_{0} B^{*}\left(t,\left(\bar{a}_{i 0}^{*}\right)^{\hat{\sigma}}(t)\right)\right] \cdot\left[-\xi_{0}^{*}\left(t, \bar{a}_{i 0}^{*}(t)\right)\right]} \\
& \quad+\left[\partial_{l} R_{j}^{*}\left(t,\left(\bar{a}_{i 0}^{*}\right){ }^{\hat{\sigma}}(t)\right) \cdot\left(a_{j}^{*}\right)^{\hat{\Delta}}(t)-\partial_{l} B^{*}\left(t,\left(\bar{a}_{i 0}^{*}\right)^{\hat{\sigma}}(t)\right)\right] \cdot\left(\xi_{l}^{*}\right)^{\hat{\sigma}}\left(t, \bar{a}_{i 0}^{*}(t)\right) \\
& \quad+R_{j}^{*}\left(t,\left(\bar{a}_{i 0}^{*}\right)^{\hat{\sigma}}(t)\right)\left(\xi_{j}^{*}\right)^{\hat{\Delta}}\left(t, \bar{a}_{i 0}^{*}(t)\right)-B^{*}\left(t,\left(\bar{a}_{i 0}^{*}\right)^{\hat{\sigma}}(t)\right) \cdot\left[-\left(\xi_{0}^{*}\right)^{\hat{\Delta}}\left(t, \bar{a}_{i 0}^{*}(t)\right)\right]=0 .
\end{aligned}
$$

From Proposition 2.2 and Remark 4.5, we get

$$
\begin{aligned}
& \partial_{0} R_{j}^{*}\left(t,\left(\bar{a}_{i 0}^{*}\right)^{\hat{\sigma}}(t)\right)=\partial_{0} R_{j}\left(-t, \bar{a}_{i 0}^{\rho}(-t)\right), \quad \partial_{0} B^{*}\left(t,\left(\bar{a}_{i 0}^{*}\right)^{\hat{\sigma}}(t)\right)=-\partial_{0} B\left(-t, \bar{a}_{i 0}^{\rho}(-t)\right), \\
& \left(\xi_{l}^{*}\right)^{\hat{\sigma}}\left(t, \bar{a}_{i 0}^{*}(t)\right)=\xi_{l}^{\rho}\left(-t, \bar{a}_{i 0}(-t)\right), \quad\left(\xi_{j}^{*}\right)^{\hat{\triangle}}\left(t, \bar{a}_{i 0}^{*}(t)\right)=-\xi_{j}^{\nabla}\left(-t, \bar{a}_{i 0}(-t)\right), \\
& \xi_{0}^{*}\left(t, \bar{a}_{i 0}^{*}(t)\right)=\xi_{0}\left(-t, \bar{a}_{i 0}(-t)\right), \quad\left(\xi_{0}^{*}\right)^{\hat{\Delta}}\left(t, \bar{a}_{i 0}^{*}(t)\right)=-\xi_{0}^{\nabla}\left(-t, \bar{a}_{i 0}(-t)\right) .
\end{aligned}
$$

Using formulae (4.6), (4.7) and (4.10), we can rewrite formula (4.9), and get the intended result.

Formula (4.8) is the Noether identity for the Birkhoffian system on time scales with nabla derivatives.

Theorem 4.9. If formula (3.1) is invariant under the infinitesimal transformations (3.8), then we obtain the following conserved quantity for the Birkhoffian system on time scales with nabla derivatives for all $\mathrm{s} \in[\mathrm{c}, \mathrm{d}]_{\mathrm{K}}$

$$
\begin{aligned}
I= & R_{j}\left(s, \bar{a}_{i 0}^{\rho}(s)\right) \cdot \xi_{j}\left(s, \bar{a}_{i 0}(s)\right)+\left\{v ( s ) \left[\partial_{0} R_{j}\left(s, \bar{a}_{i 0}^{\rho}(s)\right) \cdot a_{j}^{\nabla}(s)\right.\right. \\
& \left.\left.-\partial_{0} B\left(s, \bar{a}_{i 0}^{\rho}(s)\right)\right]-B\left(s, \bar{a}_{i 0}^{\rho}(s)\right)\right\} \cdot \xi_{0}\left(s, \bar{a}_{i 0}(s)\right)=\text { const. }
\end{aligned}
$$

Proof. From Theorem 4.3, for all $t \in[-\mathrm{d},-\mathrm{c}]^{\mathrm{k}}$, we can conclude that

$$
\begin{aligned}
I= & R_{j}^{*}\left(t,\left(\bar{a}_{i 0}^{*}\right)^{\hat{\sigma}}(t)\right) \cdot \xi_{j}^{*}\left(t, \bar{a}_{i 0}^{*}(t)\right)-\left\{\hat { \mu } ( t ) \left[\partial_{0} R_{j}^{*}\left(t,\left(\bar{a}_{i 0}^{*}\right)^{\hat{\sigma}}(t)\right) \cdot\left(a_{j}^{*}\right)^{\hat{\Delta}}(t)\right.\right. \\
& \left.\left.-\partial_{0} B^{*}\left(t,\left(\bar{a}_{i 0}^{*}\right) \hat{\sigma}(t)\right)\right]+B^{*}\left(t,\left(\bar{a}_{i 0}^{*}\right)^{\hat{\sigma}}(t)\right)\right\} \cdot\left[-\xi_{0}^{*}\left(t, \bar{a}_{i 0}^{*}(t)\right)\right],
\end{aligned}
$$

is a constant. It follows from formulae (4.6), (4.7), (4.10) and Proposition 2.2 that formula (4.11) holds.

Theorem 4.9 is the Noether theorem for the Birkhoffian system on time scales with nabla derivatives. Remark 4.10. Both methods produce the same results of Noether equations, Noether identity and conserved quantity for Birkhoffian system on time scales with nabla derivatives.

\section{An example}

Try to find the conserved quantities for the following Birkhoffian system

$$
B=\left(a_{2}^{\rho}\right)^{2}+2 t \cdot a_{2}^{\rho}, \quad R_{1}=a_{2}^{\rho}, \quad R_{2}=0 .
$$

From Theorem 4.8, we can get the following Noether identity

$$
-2 a_{2}^{\rho} \cdot \xi_{0}+\left(a_{1}^{\nabla}-2 a_{2}^{\rho}-2 t\right) \cdot \xi_{2}^{\rho}+a_{2}^{\rho} \cdot \xi_{1}^{\nabla}-\left[\left(a_{2}^{\rho}\right)^{2}+2 t \cdot a_{2}^{\rho}\right] \cdot \xi_{0}^{\nabla}=0 .
$$

By calculation, we have

$$
\xi_{0}=1, \quad \xi_{1}=2 t, \quad \xi_{2}=0 .
$$


If we consider this system on the time scale $\mathbb{T}=\left\{3^{n} \mid n \in \mathbb{N} \bigcup\{0\}\right\}$, we have

$$
\rho(t)=\frac{1}{3} t, \quad v(t)=t-\rho(t)=\frac{2}{3} t .
$$

From Theorem 4.9, we get the conserved quantity

$$
\mathrm{I}=-\frac{4}{3} \mathrm{t} \cdot \mathrm{a}_{2}^{\rho}-\left(\mathrm{a}_{2}^{\rho}\right)^{2}=\text { const. . }
$$

If we consider this system on the time scale $\mathbb{T}=h \mathbb{Z}=\{h k \mid k \in \mathbb{Z}\}, h>0$, we have

$$
\rho(t)=t-h, \quad v(t)=t-\rho(t)=h .
$$

In this case, we get the conserved quantity

$$
I=-2 h \cdot a_{2}^{\rho}-\left(a_{2}^{\rho}\right)^{2}=\text { const. . }
$$

And if we consider this system on the time scale $\mathbb{T}=\mathbb{R}$, we have

$$
\rho(t)=t, \quad v(t)=0 .
$$

In this case, we get the conserved quantity

$$
\mathrm{I}=-\mathrm{t} \cdot \mathrm{a}_{2}-\left(\mathrm{a}_{2}\right)^{2}=\text { const. . }
$$

Formula (5.1) can be found in [58].

\section{Conclusion}

Birkhoff equations, Noether identity and Noether theorem on time scales with nabla derivatives for the Birkhoffian system are obtained in this paper, where Theorem 3.3-3.7, Theorem 4.7-4.9, and Corollary 3.9 are new works. Corollary 3.8 and Corollary 3.10 coincide with the original results.

The main results are achieved through two methods: the isochronous variational principle and the duality principle. It is obtained that only when the dual results have been achieved, can the duality principle be used, though it is elegant. Therefore, the isochronous variational principle is more general.

On the basis of those results obtained in this paper, perhaps further research such as perturbation to Noether symmetry and adiabatic invariants on time scales for constrained mechanical systems can be studied. Apart from Noether symmetry method, Lie symmetry method and Mei symmetry method are also important modern integral methods. It is hoped that they can also be used to study the constrained mechanical systems on time scales.

In addition, fractional calculus has been applied to various fields of science and engineering, such as quantum mechanics, chaotic mechanics, anomalous diffusion, plasma physics and so on. Fractional mechanics can describe both conservative and non-conservative systems. Recently, fractional Euler-Lagrange equations [14], fractional Hamilton equations [12], Hamiltonian structure of fractional first order Lagrangian [38], constant of motion in fractional multi time Hamiltonian and the dual action for fractional mechanics [13], etc. have been obtained. Besides, the research on fractional calculus on time scales [19, 2123] has also just started. Therefore, studying fractional mechanical systems on time scales will be a good topic in future.

What is more, lattice fractional diffusion equations obtained through fractional differences [72, 73] have also been studied to some extent. Hence, the discrete fractional variational problem on lattices on time scales is also an aspect to study in future.

\section{Acknowledgment}

This work is supported by the National Natural Science Foundation of China (grant No. 11572212 and 11272227) and the Innovation Program for postgraduate in Higher Education Institutions of Jiangsu Province (KYLX15_0405). We would like to express our sincere thanks to the anonymous referees for their valuable comments and suggestions. 


\section{References}

[1] T. Abdeljawad, On Riemann and Caputo fractional differences, Comput. Math. Appl., 62 (2011), 1602-1611. 1

[2] T. Abdeljawad, D. Baleanu, Fractional differences and integration by parts, J. Comput. Anal. Appl., 13 (2011), 574-582. 1

[3] R. P. Agarwal, Difference equations and inequalities, Theory, methods, and applications, Second edition, Monographs and Textbooks in Pure and Applied Mathematics, Marcel Dekker, Inc., New York, (2000). 1

[4] R. Agrawal, M. Bohner, D. O'Regan, A. Peterson, Dynamic equations on time scales: a survey, Dynamic equations on time scales, J. Comput. Appl. Math., 141 (2002), 1-26. 1

[5] C. D. Ahlbrandt, M. Bohner, J. Ridenhour, Hamiltonian systems on time scales, J. Math. Anal. Appl., 250 (2000), 561-578. 1

[6] R. Almeida, D. F. M. Torres, Isoperimetric problems on time scales with nabla derivatives, J. Vib. Control, 15 (2009), 951-958. 1

[7] T. M. Atanacković, S. Konjik, S. Pilipović, S. Simić, Variational problems with fractional derivatives: invariance conditions and Noether's theorem, Nonlinear Anal., 71 (2009), 1504-1517. 1

[8] F. M. Atici, D. C. Biles, A. Lebedinsky, An application of time scales to economics, Math. Comput. Modelling, 43 (2006), 718-726. 1

[9] F. M. Atici, P. W. Eloe, Initial value problems in discrete fractional calculus, Proc. Amer. Math. Soc., 137 (2009), 981-989. 1

[10] F. M. Atici, G. S. Guseinov, On Green's functions and positive solutions for boundary value problems on time scales, Dynamic equations on time scales, J. Comput. Appl. Math., 141 (2002), 75-99. 1

[11] F. M. Atici, S. Şengül, Modeling with fractional difference equations, J. Math. Anal. Appl., 369 (2010), 1-9. 1

[12] D. Baleanu, O. P. Agrawal, Fractional Hamilton formalism within Caputo's derivative, Czechoslovak J. Phys., 56 (2006), 1087-1092. 6

[13] D. Baleanu, A. K. Golmankhaneh, A. K. Golmankhaneh, The dual action of fractional multi time Hamilton equations, Internat. J. Theoret. Phys., 48 (2009), 2558-2569. 6

[14] D. Baleanu, S. I. Muslih, Lagrangian formulation of classical fields within Riemann-Liouville fractional derivatives, Phys. Scripta, 72 (2005), 119-121. 6

[15] Z. Bartosiewicz, Ü. Kotta, E. Pawuszewicz, Equivalence of linear control systems on time scales, Proc. Estonian Acad. Sci. Phys. Math., 55 (2006), 43-52. 1

[16] Z. Bartosiewicz, N. Martins, D. F. M. Torres, The second Euler-Lagrange equation of variational calculus on time scales, Eur. J. Control, 17 (2011), 9-18.

[17] Z. Bartosiewicz, E. Pawluszewicz, Realizations of nonlinear control systems on time scales, IEEE Trans. Automat. Control, 53 (2008), 571-575. 1

[18] Z. Bartosiewicz, D. F. M. Torres, Noether's theorem on time scales, J. Math. Anal. Appl., 342 (2008), 1220-1226. 1

[19] N. R. O. Bastos, D. Mozyrska, D. F. M. Torres, Fractional derivatives and integrals on time scales via the inverse generalized Laplace transform, Int. J. Math. Comput., 11 (2011), 1-9. 6

[20] E. T. Bell, Men of mathematics, Simon and Schuster, New York, (1937). 1

[21] N. Benkhettou, A. M. B. da Cruz, D. F. M. Torres, A fractional calculus on arbitrary time scales: fractional differentiation and fractional integration, Signal Process., 107 (2015), 230-237. 6

[22] N. Benkhettou, A. Hammoudi, D.F.M. Torres, Existence and uniqueness of solution for a fractional Riemann-Liouville initial value problem on time scales, J. King Saud Univ. Sci., 28 (2016), 87-92.

[23] N. Benkhettou, S. Hassani, D.F.M. Torres, A conformable fractional calculus on arbitrary time scales, J. King Saud Univ. Sci., 28 (2016), 93-98. 6

[24] G. D. Birkhoff, Dynamical systems, With an addendum by Jurgen Moser, American Mathematical Society Colloquium Publications, American Mathematical Society, Providence, R.I., (1966). 1

[25] M. Bohner, Calculus of variations on time scales, Dynam. Systems Appl., 13 (2004), 339-349. 1

[26] M. Bohner, G. S. Guseinov, Partial differentiation on time scales, Dynam. Systems Appl., 13 (2004), 351-379. 1

[27] M. Bohner, A. Peterson, Dynamic equations on time scales. An introduction with applications, Birkhäuser Boston, Inc., Boston, MA, (2001). 1

[28] M. Bohner (Ed.), A. Peterson (Ed.), Advances in dynamic equations on time scales, Birkhäuser Boston, Inc., Boston, MA, (2003). 1

[29] P.-P. Cai, J.-L. Fu, Y.-X. Guo, Noether symmetries of the nonconservative and nonholonomic systems on time scales, Sci. China Phys. Mech. Astronom., 56 (2013), 1017-1028. 1, 3.1

[30] M. C. Caputo, Time scales: from nabla calculus to delta calculus and vice versa via duality, Int. J. Difference Equ., 5 (2010), 25-40. 1, 2, 3.3

[31] F.-L. Chen, X.-N. Luo, Y. Zhou, Existence results for nonlinear fractional difference equation, Adv. Difference Equ., 2011 (2011), 12 pages. 1

[32] X.-W. Chen, F.-X. Mei, Constrained mechanical systems and gradient systems with strong Lyapunov functions, Mech. Res. Commun., 76 (2016), 91-95. 1

[33] J. Chen, Y. Zhang, Perturbation to Noether symmetries and adiabatic invariants for Birkhoffian systems based on El-Nabulsi dynamical models, (Chinese) Acta Phys. Sin., 63 (2014), 284-290 . 1, 2 
[34] R. A. C. Ferreira, D. F. M. Torres, Higher-order calculus of variations on time scales, Mathematical control theory and finance, Springer, Berlin, (2008), 149-159. 1

[35] G. S. F. Frederico, D. F. M. Torres, A formulation of Noether's theorem for fractional problems of the calculus of variations, J. Math. Anal. Appl., 334 (2007), 834-846. 1

[36] J.-L. Fu, B.-Y. Chen, L.-Q. Chen, Noether symmetries of discrete nonholonomic dynamical systems, Phys. Lett. A, 373 (2009), 409-412. 1

[37] A. S. Galiullan, Analytical dynamics, Nauka, Moscow, (1989). 1, 3.3

[38] A. K. Golmankhaneh, A. K. Golmankhaneh, D. Baleanu, M. C. Baleanu, Hamiltonian structure of fractional first order Lagrangian, Internat. J. Theoret. Phys., 49 (2010), 365-375. 6

[39] C. S. Goodrich, On a discrete fractional three-point boundary value problem, J. Difference Equ. Appl., 18 (2012), $397-415$. 1

[40] C. S. Goodrich, A. C. Peterson, Discrete fractional calculus, Springer, Cham, (2015). 1

[41] S. Hilger, Ein Maßkettenkalkiil mit Anwendung auf Zentrumsmannigfaltigkeiten, Ph.D. thesis, Universität of Würzburg, Würzburg, (1988). 1

[42] R. Hilscher, V. Zeidan, Calculus of variations on time scales: weak local piecewise $\mathrm{C}_{\mathrm{rd}}^{1}$ solutions with variable endpoints, J. Math. Anal. Appl., 289 (2004), 143-166. 1

[43] R. Hilscher, V. Zeidan, Weak maximum principle and accessory problem for control problems on time scales, Nonlinear Anal., 70 (2009), 3209-3226. 1

[44] M. T. Holm, The Laplace transform in discrete fractional calculus, Comput. Math. Appl., 62 (2011), 1591-1601. 1

[45] L.-L. Huang, D. Baleanu, G.-C. Wu, S.-D. Zeng, A new application of the fractional Logistic map, Romanian J. Phys., 61 (2016), 1172-1179. 1

[46] S.-X. Jin, Y. Zhang, Noether symmetries for non-conservative Lagrange systems with time delay based on fractional model, Nonlinear Dynam., 79 (2015), 1169-1183. 1

[47] X.-L. Kong, H.-B. Wu, F.-X. Mei, Variational discretization of constrained Birkhoffian systems, Nonlinear Dynam., 78 (2014), 329-339. 1

[48] Z.-J. Li, S.-K. Luo, A new Lie symmetrical method of finding conserved quantity for Birkhoffian systems, Nonlinear Dynam., 70 (2012), 1117-1124.

[49] S.-K. Luo, Y.-L. Xu, Fractional Birkhoffian mechanics, Acta Mech., 226 (2015), 829-844. 1

[50] A. B. Malinowska, M. R. S. Ammi, Noether's theorem for control problems on time times, Int. J. Difference Equ., 9 (2014), 87-100. 1

[51] A. B. Malinowska, N. Martins, The second Noether theorem on time scales, Abstr. Appl. Anal. 2013 (2013), 14 pages. 1

[52] A. B. Malinowska, D. F. M. Torres, Necessary and sufficient conditions for local Pareto optimality on time scales, (Russian); translated from Sovrem. Mat. Prilozh, Optimalnoe Upravlenie, 63 (2009), 24-31, J. Math. Sci. (N.Y.) 161 (2009), 803-810. 1

[53] A. B. Malinowska, D. F. M. Torres, Strong minimizers of the calculus of variations on time scales and the Weierstrass condition, Proc. Est. Acad. Sci., 58 (2009), 205-212.

[54] N. Martins, D. F. M. Torres, Calculus of variations on time scales with nabla derivatives, Nonlinear Anal., 71 (2009), e763-e773. 1, 2.3

[55] N. Martins, D. F. M. Torres, Noether's symmetry theorem for nabla problems of the calculus of variations, Appl. Math. Lett., 23 (2010), 1432-1438. 1, 3.3

[56] F.-X. Mei, Analytical Mechanics, II, Beijing Institute of Technology Press, Beijing, (2013). 1

[57] F.-X. Mei, R.-C. Shi, Y.-F. Zhang, H.-B. Wu, Dynamics of Birkhoff Systems, Beijing University of Technology press, Beijing, (1996). 1

[58] F.-X. Mei, H.-B. Wu, Dynamics of Constrained Mechanical Systems, Beijing institute of Technology press, Beijing, (2009). 1, 5

[59] D. Mozyrska, Multiparameter fractional difference linear control systems, Discrete Dyn. Nat. Soc., 2014 (2014), 8 pages. 1

[60] D. Mozyrska, E. Pawuszewicz, Controllability of h-difference linear control systems with two fractional orders, Internat. J. Systems Sci., 46 (2015), 662-669. 1

[61] E. Noether, Invariant variation problems, Translated from the German, Nachr. Akad. Wiss. Göttingen Math.-Phys. Kl., 2 (1918), 235-257, Transport Theory Statist. Phys., 1 (1971), 186-207. 1

[62] T. Odzijewicz, A. B. Malinowska, D. F. M. Torres, Noethers theorem for fractional variational problems of variable order, Open Phys., 11 (2013), 691-701. 1

[63] K.-K. Peng, Y.-P. Luo, Dynamics symmetries of Hamiltonian system on time scales, J. Math. Phys., 55 (2014), 10 pages. 1

[64] R. M. Santilli, Foundations of theoretical mechanics, II, Birkhoffian generalization of Hamiltonian mechanics, Texts and Monographs in Physics, Springer-Verlag, New York-Berlin, (1983) 1

[65] C.-J. Song, Y. Zhang, Conserved quantities and adiabatic invariants for El-Nabulsis fractional Birkhoff system, Internat. J. Theoret. Phys., 54 (2015), 2481-2493. 1

[66] C.-J. Song, Y. Zhang, Noether theorem for Birkhoffian systems on time scales, J. Math. Phys., 56 (2015), 9 pages. 4.1

[67] C.-J. Song, Y. Zhang, Perturbation to Mei symmetry and adiabatic invariants for disturbed El-Nabulsi's fractional Birkhoff system, Commun. Theor. Phys. (Beijing), 64 (2015), 171-176. 1 
[68] H. L. Turrittin, My mathematical expectations, Symposium on Ordinary Differential Equations, Univ. Minnesota, Minneapolis, Minn., (1972); dedicated to Hugh L. Turrittin, Lecture Notes in Math., Springer, Berlin, 312 (1973), 1-22. 1

[69] G.-C. Wu, D. Baleanu, Chaos synchronization of the discrete fractional logistic map, Signal Process., 102 (2014), 96-99. 1

[70] G.-C. Wu, D. Baleanu, Discrete fractional logistic map and its chaos, Nonlinear Dynam., 75 (2014), $283-287$.

[71] G.-C. Wu, D. Baleanu, Jacobian matrix algorithm for Lyapunov exponents of the discrete fractional maps, Commun. Nonlinear Sci. Numer. Simul., 22 (2015), 95-100. 1

[72] G.-C. Wu, D. Baleanu, Z.-G. Deng, S.-D. Zeng, Lattice fractional diffusion equation in terms of a Riesz-Caputo difference, Phys. A, 438 (2015), 335-339. 6

[73] G.-C. Wu, D. Baleanu, H.-P. Xie, Riesz Riemann-Liouville difference on discrete domains, Chaos, 26 (2016), 5 pages. 1, 6

[74] G.-C. Wu, D. Baleanu, H.-P. Xie, F.-L. Chen, Chaos synchronization of fractional chaotic maps based on the stability condition, Phys. A, 460 (2016), 374-383. 1

[75] G.-C. Wu, D. Baleanu, S.-D. Zeng, Discrete chaos in fractional sine and standard maps, Phys. Lett. A, 378 (2014), 484-487. 1

[76] B. Yan, Y. Zhang, Noether's theorem for fractional Birkhoffian systems of variable order, Acta Mech., 227 (2016), 2439 2449. 1

[77] X.-H. Zhai, Y. Zhang, Noether symmetries and conserved quantities for Birkhoffian systems with time delay, Nonlinear Dynam., 77 (2014), 73-86. 1

[78] Z.-D. Zhan, W. Wei, H.-L. Xu, Hamilton-Jacobi-Bellman equations on time scales, Math. Comput. Modelling, 49 (2009), 2019-2028. 1

[79] Y. Zhang, Noether theory for Hamiltonian system on time scales, Chinese Quart. Mech., 37 (2016), 214-224. 1

[80] Y. Zhang, X.-H. Zhai, Noether symmetries and conserved quantities for fractional Birkhoffian systems, Nonlinear Dynam., 81 (2015), 469-480. 1

[81] Y. Zhang, Y. Zhou, Symmetries and conserved quantities for fractional action-like Pfaffian variational problems, Nonlinear Dynam., 73 (2013), 783-793. 1

[82] Y. Zhang, X.-S. Zhou, Noether theorem and its inverse for nonlinear dynamical systems with nonstandard Lagrangians, Nonlinear Dynam., 84 (2016), 1867-1876. 1

[83] Q.-H. Zu, J.-Q. Zhu, Noether theorem for nonholonomic nonconservative mechanical systems in phase space on time scales, J. Math. Phys., 57 (2016), 10 pages. 1 